\section{Rigorous Method to Assess Quality and Generalizability of Clinical Practice Guidelines}

Clinical practice guidelines (CPGs) are important tools for clinical decision-making in modern health care. ${ }^{1}$ The introduction of CPGs into clinical practice has revolutionized the way clinicians care for patients, by allowing them to integrate principles of evidencebased medicine with patient-specific factors and clinical judgment. ${ }^{2}$ Through dissemination of specific recommendations, CPGs attempt to standardize care according to established best practices. They also provide a means for monitoring prescriber practices at both individual and institutional levels. ${ }^{3}$ However, certain aspects of CPGs may, directly or indirectly, have negative effects on care. ${ }^{4}$ For example, use of expert opinion, lack of a stringent review process, or direct financial sponsorship may compromise the validity of a published guideline.

A recent Point Counterpoint debate in the CJHP raised many points both supporting and refuting the use of CPGs in clinical practice., ${ }^{5,6}$ With this discussion as background, our aim was to demonstrate a process of rigorous guideline appraisal by using a standardized method to assess the recently published Joint National Committee hypertension guidelines. ${ }^{\text {? }}$

The hypertension CPG was appraised using the Appraisal of Guidelines for Research and Evaluation II (AGREE II) instrument. ${ }^{8}$ Application of this tool involves ranking 23 items within 6 domains and completing additional categories for "Overall Assessment" and "Recommendation". For the 23 items and the overall assessment, the appraisers were asked to assign a rank between 1 (strongly disagree) and 7 (strongly agree). For example, the first item under the domain "scope and purpose" states, "The overall objective(s) of the guideline is (are) specifically described", and appraisers used the 7-point scale to rate how well the CPG fulfilled this criterion. Standardized domain scores were subsequently calculated according to the formula provided in AGREE II. ${ }^{8}$ Domain scores are reported as percentages, on a scale from 0 to $100 \%$, with $100 \%$ being the highest score possible. For the final recommendation, appraisers were asked to state whether or not they would recommend using the guideline, or if they would recommend using the guideline with required modifications.

The CPG was appraised independently by 6 investigators (the authors of this letter). Upon completion, all appraisals were forwarded to one investigator (K.J.W.), who reviewed and validated the data by ensuring that no item had scores differing by 6 points or more. If any item had such widely divergent scores, the guideline was redistributed to all investigators for review of the discrepant item. Once final rankings were received, standardized scores were calculated for each domain. ${ }^{8}$ The group's final recommendation was the recommendation selected most frequently by appraisers (Yes, Yes with modifications, or No).

The highest-scoring domain was clarity of presentation (90.7\%) and the lowest was applicability (34.7\%) (Table 1). The overall assessment was 75\%, and the group's final recommendation was that the CPG should be used in clinical practice, with modifications (2 appraisers recommended using the CPG as is, 4 recommended using the CPG with modifications, and none recommended not using the $\mathrm{CPG}$ ).

Although clarity of presentation, the highest-ranked domain, is an important aspect of a CPG, it does not indicate the methodologic strength of a guideline, and other domains, such as rigour of development and applicability, should be weighted more heavily. However, these results align with a previously published report that ranked highly the clarity of pharmacotherapy guidelines. ${ }^{9}$ For the hypertension guideline, the domains of applicability and stakeholder involvement were ranked lowest. The applicability domain is largely based on the provision of discussion and/or tools to facilitate implementation of the guideline in practice. Therefore, statements about facilitators of and barriers to guideline adoption, resource implications, monitoring and auditing criteria, and/or provision of advice or tools for implementation will be helpful in enhancing applicability. Although overall recommendations presented in a CPG may be generalizable to many settings, inclusion of these considerations will allow clinicians to apply the recommendations in practice. Additionally, guideline creators

\section{Table 1. Summary of Appraisal Results}

\begin{tabular}{lc} 
Domain & Scaled Domain Score (\%)* \\
\hline Scope and purpose & 88.0 \\
Stakeholder involvement & 50.0 \\
Rigour of development & 64.6 \\
Clarity of presentation & 90.7 \\
Applicability & 34.7 \\
Editorial independence & 84.7 \\
Overall guideline assessment & 75.0 \\
Overall guideline recommendation & Yes, with modifications
\end{tabular}

*Based on scoring (on a 7-point scale) by 6 appraisers, with standardized domain scores subsequently calculated according to the AGREE II formula ${ }^{8}$ and reported as percentages (highest possible score: 100\%). 
seldom seek opinions from multiple professional groups, including patients. However, such information can be valuable, as these stakeholders can offer insight into the feasibility of recommendations, which could enhance scores for both stakeholder involvement and applicability. Patient (or caregiver) perspectives are especially important, as some of the highest-quality recommendations may pose challenges for patient understanding and adherence. For these reasons, we advocate that patients or advocacy groups be included during the creation of CPGs.

In this report, we have summarized a standardized, rigorous method for appraising CPGs. This method can be adapted by those attempting to assess CPG quality and is applicable for creation of CPGs or institutional guidelines. The low scores for stakeholder involvement and applicability for the hypertension guideline appraised here, which align with previously published reports, suggest clear directions to CPG developers for ways to improve their guidelines. Clinicians can also use this information to become aware of issues pertaining to applicability and validity of CPGs for their patients.

In closing, we urge all clinicians to be aware of the limitations of CPGs and to complete their own appraisals whenever possible. Doing so will allow for better interpretation and application of evidence and will enhance the decision-making process for better patient care.

\section{References}

1. Carter A. Clinical practice guidelines. CMAJ. 1992;147(11):1649-50.

2. Davis DA, Taylor-Vaisey A. Translating guidelines into practice: a systematic review of theoretic concepts, practical experience and research evidence in the adoption of clinical practice guidelines. CMAJ. 1997;157(4):408-16.

3. Hysong SJ, Best RG, Pugh JA. Audit and feedback and clinical practice guideline adherence: making feedback actionable. Implement Sci. 2006;1:9.

4. Shaneyfelt TM, Centor RM. Reassessment of clinical practice guidelines: go gently into that good night. JAMA. 2009;301(8):868-9.
5. El Kassem W, Abdulrahman A, Raghab A, Wilbur K. Should clinical practitioners, as part of institutional or accreditation standards, be required to document their rationale when choosing to not adhere to widely accepted clinical practice guidelines? The "pro" side. Can J Hosp Pharm. 2013;66(4):253-4.

6. Elgaily DE, Sahal AO, Orabi BA, Ibrahim DA, Wilby KJ. Should clinical practitioners, as part of institutional or accreditation standards, be required to document their rationale when choosing to not adhere to widely accepted clinical practice guidelines? The "con" side. Can J Hosp Pharm. 2013;66(4):254-5.

7. James PA, Oparil S, Carter BL, Cushman WC, Dennison-Himmelfarb C, Handler J, et al. 2014 evidence-based guideline for the management of high blood pressure in adults: report from the panel members appointed to the Eighth Joint National Committee (JNC 8). JAMA. 2013;311(5):507-20.

8. Brouwers M, Kho ME, Browman GP, Burgers JS, Cluzeau F, Feder G, et al.; AGREE Next Steps Consortium. AGREE II: Advancing guideline development, reporting and evaluation in healthcare. CMAJ. 2010;182(18):e839-42.

9. Gorman SK, Chung MH, Slavik RS, Zed PJ, Wilbur K, Dhingra VK. A critical appraisal of the quality of critical care pharmacotherapy clinical practice guidelines and their strength of recommendations. Intensive Care Med. 2010;36(10): 1636-43.
Ahmed Ali Farghali, BSc(Pharm)

Raja Al-Khawaja, BSc(Pharm)

Lama Madi, BSc(Pharm)

Ahmed Elbardissy, BSc(Pharm)

Hesham Mahmoud Hamdy, BSc(Pharm)

PharmD students

Kyle J Wilby, BSP, ACPR, PharmD

Assistant Professor - Clinical Pharmacy and Practice

College of Pharmacy

Qatar University

Doha, Qatar

Competing interests: None declared.

\begin{tabular}{|c|c|c|}
\hline & Ad Page & Prescribing Information \\
\hline Baxter / Corporate & 333 & - \\
\hline Pfizer / Injectables & 326 & - \\
\hline Pfizer / Injectables & 330 & - \\
\hline Pharmaceutical Partners of Canada / Heparin & IFC & - \\
\hline Pharmaceutical Partners of Canada / Corporate & $\mathrm{OBC}$ & - \\
\hline Sanofi-Aventis / Allerject & 328,329 & - \\
\hline
\end{tabular}

